\title{
Euler-Possion 半导体模型的大时间行为
}

黄飞敏 (1), 潘荣华 (2), 于慧敏 (1)

(1) 中国科学院数学与系统科学研究院应用数学研究所, 北京 100190

(2) 乔治亚理工学院数学系, 亚特兰大, 美国

E-mail: fhuang@amt.ac.cn, panrh@math.gatech.edu, hmyu@amss.ac.cn

收稿日期: 2007-10-22；接受日期: 2007-11-26

国家自然科学基金 (批准号: 10471138), NSFC-NSAF (批准号: 10676037) 和科技部国家重点基础研究发展规划 (批 准号: 2006CB805902) 资助项目, 潘荣华受美国自然科学基金 (批准号: DMS-0505515) 资助项目

摘要本文研究了 Euler-Poisson 半导体模型弱熵解的大时间行为, 得到了一致有界的弱 熵解随着时间 $t$ 指数衰减到稳态解. 本文的结果不需要假定解是 “小”的, 也不需要假定解 的正则性.

关键词大时间行为 Euler-Possion 方程组 半导体模型 摘

$\mathrm{MSC}(2000)$ 主题分类 $35 \mathrm{M} 20,35 \mathrm{Q} 35,76 \mathrm{W05}$

\section{1 引言}

本文考虑如下的 1 维 Euler-Poisson 半导体模型:

$$
\left\{\begin{array}{l}
n_{t}+J_{x}=0, \\
J_{t}+\left(\frac{J^{2}}{n}+p(n)\right)_{x}=n E-J, \quad x \in \mathbb{R}, t>0, \\
E_{x}=n-b(x),
\end{array}\right.
$$

其中 $n \geqslant 0, J$ 和 $E$ 分别表示电子的密度, 电流强度和 (负的) 电场. $b=b(x)>0$ 称为掺杂函 数, 用来描述掺杂物质正离子的密度结构. 这里我们假设 $b(x)$ 满足

$$
\begin{aligned}
& b(x) \in C^{2}(\mathbb{R}), \quad b^{\prime}(x) \in L^{1}(\mathbb{R}) \cap H^{1}(\mathbb{R}), \\
& \lim _{x \rightarrow \pm \infty} b(x)=b^{ \pm}>0, \quad b^{*}=\sup _{x \in \mathbb{R}} b(x) \geqslant \inf _{x \in \mathbb{R}} b(x)=b_{*}>0 .
\end{aligned}
$$

$p$ 表示压力的大小, 其为一标量且满足 $\gamma$ - 律: $p(n)=n^{\gamma}(\gamma \geqslant 1)$. 该模型是用来描述亚微半导 体模型中电子的运动情形, 关于该模型的介绍及分析可参考文献 [1].

我们需要给出问题 (1) 的定解条件. 第 1 , 给出电场在 $x=-\infty$ 处的值, 即

$$
\lim _{x \rightarrow-\infty} E(x, t)=E^{-}, \quad \text { a.e. } t \in[0,+\infty),
$$


第 2 , 设 (1) 式的初始条件为

$$
n(x, 0)=n_{0}(x), \quad J(x, 0)=J_{0}(x),
$$

并且满足

$$
\lim _{x \rightarrow \pm \infty} n_{0}(x)=b^{ \pm}>0, \quad \lim _{x \rightarrow \pm \infty} J_{0}(x)=\bar{J}, \quad b_{-} E^{-}=\bar{J} .
$$

以前的工作告诉我们: 如果初始数据是在次临界区域内选取的, 则可以得到问题 (1) 和 (3)-(5) 的整体光滑解 (参见文献 $[2,3]$ ), 但是, 若初始数据是选在该区域之外, 则该问题的解 就会在有限的时间内爆破 (参见文献 [4]). 因此, 考虑问题 (1) 和 (3)-(5) 的弱熵解的存在性和 大时间形态有着很重要的意义. 文献 [5-8] 给出了整体弱摘解的存在性, 文献 $[9,10]$ 分别给出 了小初值光滑解和分片光滑解的大时间行为. 但至今还没有相关文献来考虑该模型弱摘解的 大时间行为. 关于该模型的初边值问题和非等熵的情形也有些结果, 我们可参考文献 [11-14] 及其后面的参考文献.

本文要给出问题 (1) 和 (3)-(5) 的整体弱熵解的大时间行为. 这里所得到的大时间渐近 行为定理对于所有一致有界的弱熵解都是成立的. 在证明的过程中要用到下面的机械熵 - 熵 流对:

$$
\begin{aligned}
& \eta_{e}=\frac{J^{2}}{2 n}+\frac{n^{\gamma}}{\gamma-1}, \quad q_{e}=\frac{J^{3}}{2 n^{2}}+\frac{\gamma}{\gamma-1} n^{\gamma-1} J, \quad \gamma>1 ; \\
& \eta_{e}=\frac{J^{2}}{2 n}+n \ln n, \quad q_{e}=\frac{J^{3}}{2 n^{2}}+(\ln n+1) J, \quad \gamma=1 .
\end{aligned}
$$

现在给出弱熵解的定义.

定义 1 如果有界可测函数 $(n, J, E)(x, t)$ 在分布的意义下满足方程组 (1) 和下面的熵 不等式

$$
\eta_{e t}+q_{e x}+\frac{J}{n}(J-n E) \leqslant 0,
$$

并且使得初始条件和极限限制问题 (3)- (5) 成立, 则称 $(n, J, E)(x, t)$ 为问题 (1) 和 (3)-(5) 的 弱熵解.

本文我们预设下面的条件成立:

$\mathrm{H} 1)$ 假设 $(n, J, E)(x, t)$ 为问题 (1) 和 (3)-(5) 的任一整体弱熵解并且满足

$$
0 \leqslant n(x, t) \leqslant C_{0} .
$$

在该假设下, 我们将证明: 当背景电流 $\bar{J}$ 充分小时, 上面定义的弱熵解随着时间 $t$ 指数 收玫到相应的稳态解.

\section{2 稳态方程的解}

由于方程组 (1) 中电流方程的松弛结构会起到某些阻碍的作用, 我们希望原问题的弱熵 解收敛到如下稳态方程的解:

$$
\left\{\begin{array}{l}
\tilde{J}_{x}=0 \\
\left(\frac{\tilde{J}^{2}}{\tilde{n}}+p(\tilde{n})\right)_{x}=\tilde{n} \tilde{E}-\tilde{J} \\
\tilde{E}_{x}=\tilde{n}-b(x)
\end{array}\right.
$$


其中

$$
\tilde{n}(x)-b(x) \in H^{1}(\mathbb{R}), \quad \tilde{J}=\bar{J}, \quad \tilde{E}(-\infty)=E^{-} .
$$

对问题 (9)-(10) 直接做计算 (参见文献 [10]), 得到

$$
\left[\left(\frac{p^{\prime}(\tilde{n})}{\tilde{n}}-\frac{\bar{J}^{2}}{\tilde{n}^{3}}\right) \tilde{n}_{x}\right]_{x}+\left(\frac{\bar{J}}{\tilde{n}}\right)_{x}=\tilde{n}-b(x) .
$$

这是关于 $\tilde{n}(x)$ 的一个二阶常微分方程. 显然, 严格椭圆性条件与亚音速条件 ${ }^{[15]}$

$$
p^{\prime}(\tilde{n})>\frac{\bar{J}^{2}}{\tilde{n}^{2}}
$$

等价, 其中 $p^{\prime}(\tilde{n})$ 为音速大小的平方, $\bar{J} / \tilde{n}$ 表示粒子的速度. 为保证亚音速条件 (12) 的成立, 需要

$$
\tilde{n}>\left(\frac{\bar{J}^{2}}{\gamma}\right)^{\frac{1}{(\gamma+1)}}
$$

因此假设

$\mathrm{H} 2) b(x)$ 和 $\bar{J}$ 满足 $b_{*}>\left(\bar{J}^{2} / \gamma\right)^{1 /(\gamma+1)}$.

于是, 在 H2) 的条件下, 文献 [10] 证明了如下的定理:

定理 1 假设 $b(x)$ 满足 (2) 式, 并且条件 H2) 成立, 则问题 (9)-(10) 有唯一的解 $(\tilde{n}, \bar{J}, \tilde{E})$, 满足 $b_{ \pm} \tilde{E}( \pm \infty)=\bar{J}$ 和

$$
\begin{aligned}
& b_{*} \leqslant \tilde{n}(x) \leqslant b^{*}, \quad x \in \mathbb{R}, \\
& \left|\tilde{n}(x)-b_{ \pm}\right|=O(1) e^{-c_{ \pm}|x|}, \quad \text { 当 } x \rightarrow \pm \infty, \\
& \|\tilde{n}-b\|_{H^{2}}+\sup _{x \in \mathbb{R}}\left(\left|\tilde{n}^{\prime}(x)\right|+\left|\tilde{n}^{\prime \prime}(x)\right|+|\tilde{E}(x)|\right) \leqslant C_{1},
\end{aligned}
$$

其中, $C_{1}$ 是只依赖于 $b(x)$ 的正常数, $c_{ \pm}$的形式为

$$
c_{ \pm}=\frac{\tilde{E}_{ \pm}}{p^{\prime}\left(b_{ \pm}\right)-\tilde{E}_{ \pm}^{2}} .
$$

值得指出的是, (14) 式和条件 H2) 保证了亚音速条件 (12) 成立, 于是定理 1 可以由标准 的常微分理论得出. 而文献 [9] 中运用比较原理得出了 (14) 式.

\section{3 主要结果及其证明}

本节我们要证明问题 (1) 和 (3)-(5) 的弱熵解在 $L^{2}(\mathbb{R})$ 范数下强收玫到其所对应的稳态 解, 并且给出了衰减速率——指数衰减. 为此, 我们假设

$$
y=-(E-\tilde{E}) .
$$

于是, 方程组 (1) 和 (9) 表明

$$
y_{x}=-(n-\tilde{n}), \quad y_{t}=J-\bar{J} .
$$

在本文的证明过程中, 熵不等式 (7) 起着很重要的作用. 为充分利用 (7) 式, 我们引进

$$
\begin{aligned}
& \eta_{*}=\eta_{e}-\tilde{\eta}_{e}-\nabla \tilde{\eta}_{e}(\boldsymbol{v}-\tilde{\boldsymbol{v}}), \\
& q_{*}=q_{e}-\tilde{q}_{e}-\nabla \tilde{\eta}_{e}(\boldsymbol{f}-\tilde{\boldsymbol{f}}),
\end{aligned}
$$


其中

$$
\begin{aligned}
& \tilde{\eta}_{e}=\eta_{e}(\tilde{n}, \bar{J}), \quad \tilde{q}_{e}=q_{e}(\tilde{n}, \bar{J}), \\
& \boldsymbol{v}=(n, J)^{\mathrm{T}}, \quad \boldsymbol{f}=\left(J, \frac{J^{2}}{n}+p(n)\right)^{\mathrm{T}} .
\end{aligned}
$$

以下是我们的主要结果.

定理 2 设 $(\tilde{n}, \bar{J}, \tilde{E})$ 为定理 1 所给出的稳态解. $(n, J, E)(x, t)$ 为问题 (1) 和 (3)-(5) 的 任一满足条件 H1) 的弱摘解, 并且使得

$$
y(x, 0) \in L^{2}(\mathbb{R}), \quad \int_{-\infty}^{\infty} \eta_{*}(x, 0) d x<\infty
$$

成立, 则存在适当小的正常数 $\delta$, 当 $|\bar{J}| \leqslant \delta$ 时, 存在正常数 $C$ 和 $\tilde{C}$, 使得对于任意的 $t>0$ 都 有

$$
\int_{-\infty}^{\infty}\left(y_{t}^{2}+y_{x}^{2}+y^{2}\right) d x \leqslant C e^{-\tilde{C} t} \int_{-\infty}^{\infty}\left(\eta_{*}(x, 0)+y^{2}(x, 0)\right) d x .
$$

证明 首先, 由 (17) 和 (18) 式可得到下面关于 $y$ 的方程:

$$
y_{t t}+\left(\frac{J^{2}}{n}-\frac{\bar{J}^{2}}{\tilde{n}}\right)_{x}+(p(n)-p(\tilde{n}))_{x}+y_{t}=-\tilde{n} y-\tilde{E} y_{x}+y y_{x} .
$$

将 (22) 式两边乘以 $y$ 并且在 $(-\infty,+\infty)$ 上关于 $x$ 积分, 我们得到

$$
\begin{aligned}
& \frac{d}{d t} \int_{-\infty}^{\infty}\left(y y_{t}+\frac{1}{2} y^{2}\right) d x+\int_{-\infty}^{\infty}\left(p(n)-(p(\tilde{n}))(n-\tilde{n})+\left(\tilde{n}-\frac{\tilde{E}_{x}}{2}\right) y^{2}\right) d x \\
& \quad \leqslant \int_{-\infty}^{\infty} y_{t}^{2} d x+\int_{-\infty}^{\infty}\left(\frac{J^{2}}{n}-\frac{\bar{J}^{2}}{\tilde{n}}\right) y_{x} d x .
\end{aligned}
$$

显然, 由方程组 (9) 知道

$$
\tilde{n}-\frac{\tilde{E}_{x}}{2}=\frac{1}{2}(\tilde{n}+b(x)) .
$$

同时, 注意到 (14) 式和文献 [16] 中的引理 3.1, 可得

$$
(p(n)-p(\tilde{n}))(n-\tilde{n}) \geqslant O(1)(n-\tilde{n})^{2}=O(1) y_{x}^{2} .
$$

于是, 结合 (23)- $(25)$ 式, 存在正常数 $C_{2}$, 使得

$$
\begin{gathered}
\frac{d}{d t} \int_{-\infty}^{\infty}\left(y y_{t}+\frac{1}{2} y^{2}\right) d x+C_{2} \int_{-\infty}^{\infty}\left(y_{x}^{2}+y^{2}\right) d x \\
\leqslant \int_{-\infty}^{\infty} y_{t}^{2} d x+\int_{-\infty}^{\infty}\left(\frac{J^{2}}{n}-\frac{\bar{J}^{2}}{\tilde{n}}\right) y_{x} d x .
\end{gathered}
$$

由于本文考虑的解是弱解, 并且没有关于弱解正则性的任何信息, 所以我们不能得到更 高阶的能量估计. 而仅有能量估计 (26) 式并不能得到本文的结论. 受文献 [16] 和 [17] 的启发, 我们将充分利用摘耗散的性质来达到目的.

将 (19) 式定义的 $\eta_{*}$ 和 $q_{*}$ 代入摘不等式 (7), 得到

$$
\begin{aligned}
0 \geqslant & \eta_{e t}+q_{e x}-\frac{J}{n}(n E-J) \\
= & \eta_{* t}+q_{* x}+\nabla \tilde{\eta}_{e}(\boldsymbol{v}-\tilde{\boldsymbol{v}})_{t}+\nabla \tilde{\eta}_{e}(\boldsymbol{f}-\tilde{\boldsymbol{f}})_{x} \\
& +\tilde{q}_{e x}+\left(\nabla \tilde{\eta}_{e}\right)_{x}(\boldsymbol{f}-\tilde{\boldsymbol{f}})-\frac{J}{n}(n E-J) .
\end{aligned}
$$


简化 (27) 式中的诸项. 由 (17)、(18) 和 (22) 式可得到下面两式

$$
\nabla \tilde{\eta}_{e}(\boldsymbol{v}-\tilde{\boldsymbol{v}})_{t}+\nabla \tilde{\eta}_{e}(\boldsymbol{f}-\tilde{\boldsymbol{f}})_{x}=\frac{\bar{J}}{\tilde{n}}\left(n E-\tilde{n} \tilde{E}-y_{t}\right),
$$

和

$$
\begin{aligned}
\tilde{q}_{e x} & +\left(\nabla \tilde{\eta}_{e}\right)_{x}(\boldsymbol{f}-\tilde{\boldsymbol{f}}) \\
& =\left[\gamma \tilde{n}^{\gamma-2} J+\frac{\bar{J}^{2}}{\tilde{n}^{3}} J-2 \frac{\bar{J}^{3}}{\tilde{n}^{3}}-\frac{\bar{J}}{\tilde{n}^{2}}\left(\frac{J^{2}}{n}-\frac{\bar{J}^{2}}{\tilde{n}}+p(n)-p(\tilde{n})\right)\right] \tilde{n}_{x} .
\end{aligned}
$$

于是, 由 (27) 和 (28) 式可得

$$
\eta_{* t}+q_{* x} \leqslant J E-\frac{J^{2}}{n}-\frac{\bar{J}}{\tilde{n}}\left(n E-\tilde{n} \tilde{E}-y_{t}\right)-\left[\tilde{q}_{e x}+\left(\nabla \tilde{\eta}_{e}\right)_{x}(\boldsymbol{f}-\tilde{\boldsymbol{f}})\right] .
$$

更进一步, 注意到

$$
\tilde{E}=\frac{1}{\tilde{n}}\left(\frac{\bar{J}^{2}}{\tilde{n}}+p(\tilde{n})\right)_{x}+\frac{\bar{J}}{\tilde{n}}=\gamma \tilde{n}^{\gamma-2} \tilde{n}_{x}-\frac{\bar{J}^{2}}{\tilde{n}^{3}} \tilde{n}_{x}+\frac{\bar{J}}{\tilde{n}}
$$

有

$$
\begin{aligned}
J E & =J \tilde{E}-y y_{t}-y \bar{J} \\
& =-\frac{\bar{J}^{2}}{\tilde{n}^{3}} J \tilde{n}_{x}+\gamma \tilde{n}^{\gamma-2} J \tilde{n}_{x}+\frac{\bar{J}}{\tilde{n}} y_{t}+\frac{\bar{J}^{2}}{\tilde{n}}-y y_{t}-y \bar{J} .
\end{aligned}
$$

另一方面,

$$
\begin{aligned}
-\frac{\bar{J}}{\tilde{n}}\left(n E-\tilde{n} \tilde{E}-y_{t}\right) & =-\frac{\bar{J}}{\tilde{n}}\left(y y_{x}-\tilde{E} y_{x}-y \tilde{n}-y_{t}\right) \\
& =-\frac{\bar{J}^{3}}{\tilde{n}^{4}} y_{x} \tilde{n}_{x}+\gamma \tilde{n}^{\gamma-3} \bar{J} y_{x} \tilde{n}_{x}+\frac{\bar{J}^{2}}{\tilde{n}^{2}} y_{x}-\frac{\bar{J}}{\tilde{n}} y y_{x}+\bar{J} y+\frac{\bar{J}}{\tilde{n}} y_{t} .
\end{aligned}
$$

由 (29)-(32) 经过简单的计算可得

$$
\left(\eta_{*}+\frac{1}{2} y^{2}\right)_{t}+q_{* x}+Q_{1} \leqslant \frac{\bar{J} \tilde{n}_{x}}{\tilde{n}^{2}}\left(Q_{1}+Q_{2}-\frac{1}{2} y^{2}\right)-\left(\frac{\bar{J}}{2 \tilde{n}} y^{2}\right)_{x},
$$

其中

$$
Q_{1}=\frac{J^{2}}{n}-\frac{\bar{J}^{2}}{\tilde{n}}-\frac{2 \bar{J}}{\tilde{n}} y_{t}-\frac{\bar{J}^{2}}{\tilde{n}^{2}} y_{x}, \quad Q_{2}=n^{\gamma}-\tilde{n}^{\gamma}+\gamma \tilde{n}^{\gamma-1} y_{x}
$$

显然, $Q_{1}$ 是 $J^{2} / n$ 在 $(\tilde{n}, \bar{J})$ 处做 Taylor 展开的二阶余项, 而 $Q_{2}$ 是压力大小 $n^{\gamma}$ 在 $\tilde{n}$ 处做相 应展开的余项. 当 $\gamma=1$ 时 $Q_{2}=0$. 由 $J^{2} / n$ 和 $n^{\gamma}$ 的凸性可知 $Q_{1}$ 和 $Q_{2}$ 是非负的. 对 (33) 式在 $(-\infty,+\infty)$ 上关于 $x$ 积分, 可得

$$
\frac{d}{d t} \int_{-\infty}^{\infty}\left(\eta_{*}+\frac{1}{2} y^{2}\right) d x+\int_{-\infty}^{\infty} Q_{1} d x \leqslant \int_{-\infty}^{\infty} \frac{\bar{J} \tilde{n}_{x}}{\tilde{n}^{2}}\left(Q_{1}+Q_{2}-\frac{1}{2} y^{2}\right) d x
$$

设 $\Lambda=\max \left\{b^{*}, C_{0}\right\}$, 其中 $C_{0}$ 如 H1) 中给出. 对 (35) 式两边乘以 $\lambda=2 \Lambda+1$ 并将所得 结果与 (23) 式相加, 得到

$$
\frac{d}{d t} \int_{-\infty}^{\infty} F_{1} d x+\int_{-\infty}^{\infty}\left(F_{2}+F_{3}+F_{4}\right) d x \leqslant 0
$$


其中

$$
\begin{aligned}
& F_{1}=\lambda \eta_{*}+\frac{1}{2}(1+\lambda) y^{2}+y y_{t}, \\
& F_{2}=\lambda\left(1-\frac{\bar{J} \tilde{n}_{x}}{\tilde{n}^{2}}\right) Q_{1}-y_{t}^{2}-\left(\frac{J^{2}}{n}-\frac{\bar{J}^{2}}{\tilde{n}}\right) y_{x}, \\
& F_{3}=(p(n)-p(\tilde{n}))(n-\tilde{n})-\lambda \frac{\bar{J} \tilde{n}_{x}}{\tilde{n}^{2}} Q_{2}, \\
& F_{4}=\left[\left(\tilde{n}-\frac{\tilde{E}_{x}}{2}\right)+\frac{1}{2} \lambda \frac{\bar{J} \tilde{n}_{x}}{\tilde{n}^{2}}\right] y^{2} .
\end{aligned}
$$

由定理 1 知, 存在 $C_{3}>0$, 满足

$$
\left|\frac{\bar{J} \tilde{n}_{x}}{\tilde{n}^{2}}\right| \leqslant C_{3} \delta
$$

注意到 (14) 和 (24) 式, 我们可对 $F_{4}$ 做如下的估计:

$$
F_{4} \geqslant \frac{1}{2}\left(2 b_{*}-\lambda C_{3} \delta\right) y^{2} .
$$

当 $\gamma=1$ 时 $Q_{2}=0$, 于是 $F_{3}=y_{x}^{2}$. 当 $\gamma>1$ 时文献 [18] 中的引理 5.2 保证存在一正常数 $C_{4}$, 使得

$$
Q_{2} \leqslant C_{4}(p(n)-p(\tilde{n}))(n-\tilde{n})
$$

于是

$$
F_{3} \geqslant\left(1-C_{3} C_{4} \lambda \delta\right)(p(n)-p(\tilde{n}))(n-\tilde{n})
$$

现在讨论 $F_{2}$. 由 $Q_{1}$ 的定义知

$$
\left(\frac{J^{2}}{n}-\frac{\bar{J}^{2}}{\tilde{n}}\right) y_{x}=y_{x} Q_{1}+\frac{2 \bar{J}}{\tilde{n}} y_{t} y_{x}+\frac{\bar{J}^{2}}{\tilde{n}^{2}} y_{x}^{2} .
$$

另一方面, 由

$$
\begin{aligned}
Q_{1} & =\frac{J^{2}}{n}-\frac{J^{2}}{\tilde{n}}+\frac{J^{2}}{\tilde{n}}-\frac{\bar{J}^{2}}{\tilde{n}}-\frac{2 \bar{J}}{\tilde{n}} y_{t}-\frac{\bar{J}^{2}}{\tilde{n}^{2}} y_{x} \\
& =\frac{y_{t}^{2}}{\tilde{n}}+\frac{y_{x}}{\tilde{n}}\left(\frac{J^{2}}{n}-\frac{\bar{J}^{2}}{\tilde{n}}\right) \\
& =\frac{y_{t}^{2}}{\tilde{n}}+\frac{y_{x}}{\tilde{n}}\left(Q_{1}+\frac{2 \bar{J}}{\tilde{n}} y_{t}+\frac{\bar{J}^{2}}{\tilde{n}^{2}} y_{x}\right),
\end{aligned}
$$

可得

$$
\frac{n}{\tilde{n}} Q_{1}=\frac{y_{t}^{2}}{\tilde{n}}+\frac{2 \bar{J}}{\tilde{n}^{2}} y_{t} y_{x}+\frac{\bar{J}^{2}}{\tilde{n}^{3}} y_{x}^{2}
$$

从而有

$$
\left\{\begin{array}{l}
Q_{1} \geqslant \frac{y_{t}^{2}}{\tilde{n}}+\frac{2 \bar{J}}{\tilde{n}^{2}} y_{t} y_{x}, \quad n \leqslant \tilde{n} ; \\
Q_{1} \geqslant \frac{\tilde{n}}{n}\left(\frac{y_{t}^{2}}{\tilde{n}}+\frac{2 \bar{J}}{\tilde{n}^{2}} y_{t} y_{x}\right), \quad n>\tilde{n} .
\end{array}\right.
$$

我们取 $\delta<\frac{1}{2}\left(C_{3} \lambda\right)^{-1}$, 这意味着 $C_{3} \lambda \delta<\frac{1}{2}$. 由 $\left|y_{x}\right| \leqslant \Lambda$ 及 (40) 和 (42) 式知存在两个正常数 $C_{5}$ 和 $C_{6}$, 使得

$$
F_{2} \geqslant\left[\lambda\left(1-C_{3} \delta\right)-y_{x}\right] Q_{1}-y_{t}^{2}-\frac{2 \bar{J}}{\tilde{n}} y_{t} y_{x}-\frac{\bar{J}^{2}}{\tilde{n}^{2}} y_{x}^{2}
$$




$$
\geqslant C_{5} Q_{1}-C_{6} \delta y_{x}^{2}
$$

由 (38) 和 (39) 及 $(43)$ 式, 知道对于适当小的 $\delta$ 一定存在 $C_{7}>0$, 使得

$$
F_{2}+F_{3}+F_{4} \geqslant C_{7}\left(Q_{1}+y_{x}^{2}+y^{2}\right) \text {. }
$$

下面讨论 $F_{1}$. 显然

$$
\eta_{*}=\frac{Q_{1}}{2}+\frac{1}{\gamma-1} Q_{2}, \quad \gamma>1 ; \quad \eta_{*}=\frac{Q_{1}}{2}+Q_{3}, \quad \gamma=1,
$$

其中

$$
Q_{3}=n \ln n-\tilde{n} \ln \tilde{n}-(\ln \tilde{n}+1)(n-\tilde{n}) .
$$

由文献 [17] 中的引理 3.1 知道存在 $C_{8}>0$ 满足

$$
Q_{2} \geqslant C_{8} y_{x}^{2} \text {. }
$$

由于 $Q_{3}$ 是凸函数 $n \ln n$ 在 $\tilde{n} \geqslant b_{*}>0$ 处 Taylor 展开的二阶余项, 利用凸函数的性质和 $n$ 的 有界性, 显然存在 $C_{9}>0$ 和 $C_{10}>0$, 使得

$$
C_{9} y_{x}^{2} \leqslant Q_{3} \leqslant C_{10} y_{x}^{2} .
$$

由 (37)、(42) 和 (45)-(48) 式及 $\delta$ 的小性可找到 $C_{11}>0$ 和 $C_{12}>0$, 使得

$$
C_{11}\left(y^{2}+y_{x}^{2}+y_{t}^{2}\right) \leqslant C_{12}\left(y^{2}+y_{x}^{2}+Q_{1}\right) \leqslant F_{1} \leqslant C_{13}\left(y^{2}+y_{x}^{2}+Q_{1}\right) .
$$

于是 (36)、(44) 和 (49) 式表明

$$
\frac{d}{d t} \int_{-\infty}^{\infty} F_{1} d x+\frac{C_{7}}{C_{13}} \int_{-\infty}^{\infty} F_{1} d x \leqslant 0
$$

结合 (49) 式我们得出 (21) 式. 至此完成了定理的证明.

\section{4 一些说明}

在这一部分中, 我们要对假设、证明及其结论做出一些注释.

1) 在定理 2 的证明中, 我们利用了 $y( \pm \infty, t)=0$ 这一事实. 事实上, 初始状态表明 $y( \pm \infty, 0)=0,(21)$ 式及标准的连续性讨论即可证明这一点.

2 ) 在定理 2 中要求 $|\bar{J}|$ 适当小的同时可保证亚音速条件 (12) 成立. 在本文的证明过程 中, 若要求背景速度 $|\bar{J}| / b_{*}$ 适当小, 则定理 2 仍然成立. 值得指出的是, 在定理 2 中我们并没 有要求 $\delta$ 是任意的小. 事实上, 可以用 $b_{*}, b^{*}, C_{0}$ 及出现在证明中的一些凸函数来表示 $\delta$ 的上 界, 这是一些烦琐的直接计算, 而不是本文的主要目的. 但是, 如果去掉关于 $\bar{J}$ 或者 $\bar{J} / b_{*}$ 的 限制, 我们还不清楚 (21) 式是否成立.

3) 密度的一致上界估计在我们的证明过程中起着非常重要的作用. 但是, 已知的结果告 诉我们: 方程组 (1) 的 $L^{\infty}$ 弱熵解的界一般都与时间 $t$ 有关 (参见文献 [5,6,8]). 如何证明 方程组 (1) 的 $L^{\infty}$ 弱熵解 (特别是密度) 的一致有界性还是一个公开问题. 然而, 在文献 [10] 中构造的逐段光滑解和文献 [3] 中的次临界的整体光滑解都具有形如 (8) 式的一致有界性质. 因此, 定理 2 对文献 $[3,10]$ 中所得到的解成立.

致谢感谢审稿人提出的宝贵意见. 


\section{参考文献}

1 Markowich P, Ringhofer C, Schmeiser C. Semiconductors Equations. New York: Springer-Verlag, 1990

2 Ali G. Global existence of smooth solutions of the N-dimensional Euler-Poisson model. SIAM J Math Anal, 35: 389-422 (2003)

3 Tadmor E, Wei D. On the global regularity of sub-critical Euler-Poisson equations with pressure. Preprint, 2007

4 Chen G Q, Wang D H. Formation of singularity in compressible Euler-Poisson fluids with heat diffusion and damping relaxation. J Differential Equations, 144: 44-65 (1998)

5 Marcati P, Natalini R. Weak solutions to a hydrodynamic model for semiconductors: the Cauchy problem. Proc Roy Soc Edinburgh Sect A, 125: 115-131 (1995)

6 Poupaud F, Rascle M, Vila J P. Global solutions to the isothermal Euler-Poisson system with arbitrarily large data. J Differential Equations, 123: 93-121 (1995)

7 Wang D H, Wang Z J. Large BV solutions to the compressible isothermal Euler-Poisson equations with spherical symmetry. Nonlinearity, 19: 1985-2004 (2006)

8 Huang F M, Li T H, Yu H M. Weak solution to isothermal hydrodynamic model for semiconductors. Preprint, 2007

9 Luo T, Natalini R, Xin Z P. Large time behavior of the solutions to a hydrodynamic model for semiconsuctors. SIAM J Appl Math, 59: 810-830 (1998)

10 Li H L, Markowich P, Mei M. Asymptotic behavior of subsonic entropy solutions of isentropic Euler-Poisson equations. Quart Appl Math, 60: 773-796 (2002)

11 Li H L, Markowich P, Mei M. Asymptotic behavior of solutions of the hydrodynamic model of semiconductors. Proc Ray Soc Edinburgh Sect A-Math, 132: 359-378 (2002)

12 Matsumura A, Murakami T. Asymptotic behavior of solutions for a fluid dynamical model of semiconductor equation. In: Research Institute of Mathematical Sciences, Vol 1495, Kokyuroku, 2005, 60-70

13 Guo Y, Strauss W. Stability of semiconductor states with insulating and contact boundary conditions. Arch Rational Mech Anal, 179: 1-30 (2005)

14 Hattori H, Zhu C. Asymptotic behavior of the solutions to a non-isentropic hydrodynamic model of semiconductors. J Differential Equations, 144: 353-389 (1998)

15 Gamba I. Stationary transonic solutions of a one-dimensional hydrodynamic model for semiconductor. Comm Partial Differential Equations, 17: 553-577 (1992)

16 Huang F, Pan R. Convergence rate for compressible Euler Equations with damping and vacuum. Arch Ration Mech Anal, 166: 359-376 (2003)

17 Huang F, Marcati P, Pan R. Convergence to Barenblatt solution for the compressible Euler equations with damping and vacuum. Arch Ration Mech Anal, 176: 1-24 (2005)

18 Pan R, Zhao K. Initial Boundary value problem for compressible Euler equations with damping. Indiana Univ Math J, In press 